\section{Versuchsanordnung}

Zu den Vorschriften ${ }^{3}$ über die Manometrie der Körperzellen ist hier lediglich hinzuzufügen, daß das aktive Ascitesserum besonders sorgfältig durch Waschen mit inaktiviertem Serum entfernt werden muß, da aktives Serum Katalase enthalten kann. 10 Min. Erhitzen auf $57^{\circ}$ zerstört die Blutkatalase.

\section{Protokoll}

\section{$\mathrm{H}_{2} \mathrm{O}_{2}$-Zersetzung durch Ascites-Krebszellen und durch Chorion der Maus}

Ascites-Krebszellen ( $=2 \mathrm{mg}$ Trockensubstanz) wurden zentrifugiert, 2-mal mit inaktiviertem AscitesSerum $\left(10^{\prime} 57^{\circ}\right)$ gewaschen und auf $3 \mathrm{~cm}^{3}$ mit inaktiviertem Ascites-Serum aufgefüllt.

Chorion von Mäuseembryonen wurde 2-mal mit inaktiviertem Ascites-Serum gewaschen und dann in $3 \mathrm{~cm}^{3}$ inaktiviertem Ascites-Serum suspendiert. Gewicht pro Gefäß 2,6 mg.

Anordnung: Glucose wurde nicht zugesetzt. Von der mit $\mathrm{KMnO}_{4}$ titrierten $\mathrm{H}_{2} \mathrm{O}_{2}$-Lösung wurden $0,2 \mathrm{~cm}^{3}$ $=15,5 \mu$ Mole in die Ansatzbirne eines ManometrieKegels gegeben, dessen Hauptraum die Zellen, suspendiert in $3 \mathrm{~cm}^{3}$ inaktiviertem Ascites-Serum, enthielt. Der Gasraum enthielt 5\% $\mathrm{CO}_{2}$-Luft. Die Temperatur war $38^{\circ}$. Die folgenden Druckänderungen wurden nach Zugabe des $\mathrm{H}_{2} \mathrm{O}_{2}$ erhalten:

\begin{tabular}{|c|c|c|c|c|}
\hline \multirow[b]{2}{*}[\text{Min.}]{} & \multicolumn{2}{|c|}{ Chorion } & \multicolumn{2}{|c|}{ Krebszellen } \\
\hline & {$[\overline{\mathrm{mm}}]$} & $\underset{[\mathrm{mm}]}{+\mathrm{H}_{2} \mathrm{O}_{2}}$ & {$[\overline{\mathrm{mm}}]$} & $\underset{[\mathrm{mm}]}{+\mathrm{H}_{2} \mathrm{O}_{2}}$ \\
\hline $\begin{array}{r}2 \\
4 \\
6 \\
8 \\
10 \\
15 \\
20 \\
30 \\
45 \\
60\end{array}$ & $\begin{array}{ll} & 0 \\
& 0 \\
- & 0,5 \\
- & 0,5 \\
- & 1,0 \\
- & 2,5 \\
- & 3,5 \\
- & 5,5 \\
-\quad 9,0 \\
-\quad 12,5\end{array}$ & $\begin{array}{l}+\quad 14 \\
+\quad 40 \\
+\quad 61,5 \\
+\quad 76,5 \\
+\quad 87,0 \\
+103,5 \\
+112,5 \\
+120,0 \\
+121,5 \\
+121,5\end{array}$ & $\begin{array}{c}-0,5 \\
+0,5 \\
0 \\
0 \\
-0,5 \\
-0,5 \\
-2,0 \\
-1,5 \\
-4,0 \\
-6,0\end{array}$ & $\begin{aligned} & 0 \\
&+\quad 4,5 \\
&+\quad 8,5 \\
&+11,5 \\
&+13,5 \\
&+17,0 \\
&+21,0 \\
&+27,0 \\
&+33,5 \\
&+37,0\end{aligned}$ \\
\hline 60 & \multicolumn{2}{|c|}{$\begin{array}{l}X_{0_{2}}=121,5 \cdot 1,38 \\
\quad=168 \mathrm{~mm}^{3} \\
\quad=7,5 \mu \text { Mole } \\
\mathrm{O}_{2}, \text { während } 15,5 \mu \\
\text { Mole } \mathrm{H}_{2} \mathrm{O}_{2}=7,75 \mu \\
\text { Mole } \mathrm{O}_{2} \text { zugegeben } \\
\text { worden waren. Aus- } \\
\text { beute an } \mathrm{O}_{2}=97 \\
\text { Prozent. }\end{array}$} & \multicolumn{2}{|c|}{$\begin{array}{l}\begin{array}{l}X_{0_{2}}=37 \cdot 1,39 \\
\quad=51,5 \mathrm{~mm}^{3}\end{array} \\
\quad=2,3 \mu \text { Mole } \\
\mathrm{O}_{2}, \text { während } 15,5 \mu \\
\text { Mole } \mathrm{H}_{2} \mathrm{O}_{2}=7,75 \mu \\
\text { Mole } \mathrm{O}_{2} \text { zugegeben } \\
\text { worden waren. Aus- } \\
\text { beute an } \mathrm{O}_{2}=30 \\
\text { Prozent. }\end{array}$} \\
\hline
\end{tabular}

Tab. 1. Beobachtete Druckänderungen bei Zusatz von $\mathrm{H}_{2} \mathrm{O}_{2}$.

Zur Messung des Stoffwechsels so vorbehandelter Zellen wurden $2 \mathrm{mg}$ Glucose $/ \mathrm{cm}^{3}$ zugesetzt. Milchsäure wurde zur Stoffwechselmessung nicht zugesetzt, da Asciteskrebs-Serum viel Milchsäure enthält.

\title{
Untersuchungen zur Charakterisierung des JHM-Virus
}

\section{Ultrafiltration und Sedimentation}

\author{
Von Oskar Drees \\ Aus dem Laboratorium der Stiftung zur Erforschung der spinalen Kinderlähmung und der \\ Multiplen Sklerose, Hamburg-Eppendorf \\ (Z. Naturforschg. 12 b, 395-400 [1957]; eingegangen am 18. Oktober 1956)
}

\begin{abstract}
Es wird über weitere Untersuchungen zur Charakterisierung des sogenannten JHM-Virus berichtet.

Ultrafiltrations-Versuche mit infektiösen Mäusegehirn-Homogenaten durch Membranfilter von abgestufter Porenweite lieferten eine Filtrationskurve, deren Verlauf eine homogene Größenverteilung des infektiösen Prinzips anzeigt. Der Filtrations-Endpunkt wurde zu etwa $175 \mathrm{~m} \mu$ mittlerer Porenweite bestimmt. Unter der Annahme kugelförmiger Teilchen ergibt sich daraus eine Größenordnung des Virus von 90 bis $130 \mathrm{~m} \mu$ Durchmesser.

Weiterhin wurde die Sedimentations-Konstante des Virus auf biologischem Wege bestimmt. Zwei bei unterschiedlichen Drehzahlen durchgeführte Versuchsreihen führten zu Mittelwerten von $969 \mathrm{~S}$ und $1214 \mathrm{~S}$.

Aus den Sedimentations-Konstanten ergibt sich, wenn die experimentell vorerst nicht bestimmbare Dichte des Virus zwischen 1,1 und 1,3 angenommen wird, ein Teilchen-Durchmesser von 77 bis $135 \mathrm{~m} \mu$ (im Falle $\left.s_{20}=969\right)$ bzw. von 86 bis $152 \mathrm{~m} \mu$ (im Falle $\left.s_{20}=1214\right)$.
\end{abstract}

In einer vorangegangenen Mitt. ${ }^{1}$ berichteten wir über die $p_{\mathrm{H}}$-Resistenz und partielle Reinigung des

1 O. Drees, Z. Naturforschg. 10 b, 332 [1955].

2 F. S. Cheever, J. B. Daniels, A. M. Pappenheimer u. O. T. Balley, J. exp. Medicine 90, 181, 195 [1949]. von Cheever et al. ${ }^{2}$ aus Mäusen isolierten und später von Kersting und Petre ${ }^{3}$ auf Affen übertrage-

3 G. Kersting u. E. Pette, Dtsch. Z. Nervenheilkunde 174, 283 [1956]. 
nen JHM-Virus ${ }^{*}$. Zur näheren Charakterisierung dieses für neuropathologische Fragestellungen interessanten Erregers erschien uns die Kenntnis morphologischer und physikalisch-chemischer Eigenschaften wünschenswert. Leider gelang es uns bisher nicht, hochgereinigte und vor allem ausreichend konzentrierte Viruspräparate darzustellen, die eine sichere elektronenoptische Identifizierung des Erregers und ein exaktes Studium seiner physikalischen Eigenschaften gestatten. Wir haben uns deshalb vorerst auf Ultrafiltrations-Versuche und eine biologische Bestimmung der Sedimentations-Konstante beschränken müssen. Die Ergebnisse dieser Untersuchungen, die als Charakterisierung des JHM-Virus in erster Annäherung zu werten sind, seien in der vorliegenden Arbeit mitgeteilt.

\section{Material und Methoden}

\section{a) Virusstamm, Materialgewinnung und biologischer Test}

Die für die folgenden Untersuchungen verwendete Virus-Suspension stellten wir aus Gehirnen und Rükkenmark erkrankter Mäuse her, die bei der 8. der in unserem Laboratorium durchgeführten Mauspassagen anfielen. Einzelheiten über den Virusstamm wurden ebenso wie die Technik der Materialgewinnung und des biologischen Tests bereits in der ersten Mitt. ${ }^{1}$ beschrieben. Ergänzt sei, da $\beta$ bei den Titerbestimmungen der $\log$ des Faktors der Verdünnungsreihen 0,5 betrug.

\section{b) Ultrafiltration}

Wir arbeiteten mit handelsüblichen Membranfiltern * nach Zsigmondi ${ }^{4}$. Die mittleren Porenweiten betrugen $600,400,300,275,250,200$ und $175 \mathrm{~m} \mu$. Die Filtration erfolgte in Mikrofiltrations-Geräten aus Glas* unter 2 Atm. Überdruck.

Für die Filtrations-Versuche wurde die Virus-Stammsuspension (10-proz. wässeriges Gehirn-RückenmarkHomogenat) $1: 10$ weiterverdünnt und durch Zentrifu. gation (10 Min. bei $10000 \mathrm{U} / \mathrm{Min}$.) bis zur mäßigen Opaleszens geklärt. Als Verdünnungsmittel diente eine wässerige Lösung von 2,5\% „Heart Infusion Broth“ $(\text { DIFCO })^{* *}$ und $0,2 \%$ "Neopeptone“ (DIFCO) **, $p_{\mathrm{H}} 7,4$. Durch diesen Zusatz, den wir an Stelle der von früheren Autoren verwendeten „HARTLEYS Broth“ 5 wählten, sollten eventuelle Adsorptions-Erscheinungen in den Filtern vermieden bzw. herabgesetzt werden ${ }^{6}$.

* Von Herrn Prof. Dalldorf (Albany, USA.) erfuhren wir, daß die Bezeichnung "JHM“ in Gedenken des verstorbenen Dr. J. Howard MǘlLer gewählt wurde.

* Hersteller: Membranfilter-Gesellschaft, Göttingen.

${ }^{4}$ R. Zsigmondi u. W. Bachmann, Z. anorg. allg. Chem. 103, 119 [1918].

** Hersteller: DIFCO Laboratories, Detroit, Michigan, USA.

5 P. Hartley, J. Pathol. Bacteriol. 25, 479 [1922].
Für Filtrationen durch Membranen von weniger als $600 \mathrm{~m} \mu$ mittlerer Porenweite dienten stets Viruslösungen (sog. Stammfiltrate), die bei $600 \mathrm{~m} \mu$ vorfiltriert waren.

\section{c) Ultrazentrifugation}

Die Sedimentations-Versuche führten wir im analytischen Rotor der luftgetriebenen Phywe-Ultrazentrifuge durch. Nach beendeter Zentrifugation wurde der Uberstand vorsichtig abpipettiert, durchmischt und auf seine Infektiosität hin geprüft. Aus der Aktivitätsabnahme im Vergleich zum Ausgangsmaterial läßt sich die Sedimentations-Konstante berechnen (Näheres s. S. 398). Um ein Aufwirbeln des abgeschleuderten Virus beim $\mathrm{Ab}$ bremsen der Zentrifuge und Herausnehmen des Überstandes zu verhindern, wurde der Boden der sektorförmigen Zelle nach dem Prinzip der „improvisierten Separationszelle ${ }^{7,8}$ mit einer doppelten Lage dicken Filtrierpapieres bedeckt.

Während des Zentrifugierens wurde die Temperatur des Rotors durch zirkulierende Kältesole auf 14 bis $16^{\circ} \mathrm{C}$ begrenzt. Bei entsprechender Vorkühlung des Rotors und des Zentrifugen-Gehäuses betrugen die Temperatur-Schwankungen von Beginn bis Ende eines Laufes weniger als $\pm 0,5^{\circ} \mathrm{C}$.

Die Entfernung zwischen Drehzentrum und innerer Zellkante ist mit $5,81 \mathrm{~cm}$ festgelegt. Die für die Auswertung wichtigen Abstände zwischen Drehzentrum und Meniskus sowie Drehzentrum und Zellenboden (Filtrierpapier-Lage) wurden jeweils optisch bestimmt.

\section{Ergebnisse}

\section{Filtrations-Versuche}

\section{a) Methodische Vorversuche}

Zahlreiche Untersuchungen mit den von Elford eingeführten und seither bei Größenbestimmungen von Viren meistverwendeten "gradocol membranes" 6,9 haben gezeigt, daß folgende Fehlerquellen oft einen zu hohen Filtrations-Endpunkt und somit falsche Vorstellungen von den Dimensionen der filtrierten Viruspartikel liefern können:

1. Adsorptions-Erscheinungen in den Filtern, vor allem zu Beginn des Filtrationsvorganges,

2. mechanische Verstopfungen durch virusfremde Begleitstoffe,

3. niedrige Infektionstiter der verwendeten VirusSuspensionen.

6 W. J. Elford, in: Handbuch der Virusforschung, Bd. I, herausgeg. v. R. Doerr u. C. Hallauer, Julius Springer, Wien 1938.

7 R. Markнam, in: Progress in Biophysics and Biophysical Chemistry, Bd. 3, London 1953.

8 O. Drees, erscheint demnächst in Kolloid-Z.

9 W. J. Elford, J. Pathol. Bacteriol. 34, 505 [1931]. 
Um normale Filtrationskurven und annähernd „wahre“ Filtrations-Endpunkte zu erhalten, ist es notwendig, jedes Versuchssystem auf die genannten störenden Faktoren hin zu überprüfen. Für unsere Experimente erschien uns dies um so wichtiger, als mit Membranfiltern bislang nur vereinzelt Größenbestimmungen an Viren oder ähnlich dimensionierten Teilchen durchgeführt wurden ${ }^{10-12}$.

Da der Titer $\left(L D_{50}\right)$ unserer Virus-Suspension etwa $10^{-5}$ betrug, hielten wir es zwecks Ausschaltung der unter Punkt 3 aufgeführten Fehlerquelle für angebracht, eine Endverdünnung der zu filtrierenden Virus-Gehirn-Homogenate von $10^{-2}$ nicht zu überschreiten. Bei der Filtration 1-proz., durch Zentrifugieren und Vorfiltrieren $(600 \mathrm{~m} \mu)$ bis zur schwachen Opaleszens geklärter Suspensionen zeigte sich jedoch für Filter unter $300 \mathrm{~m} \mu$ mittlerer Porenweite eine auffallend rasche Abnahme der Filtrations-Geschwindigkeit (Abb. 1). Nach mehr als 20 bis 30 Min. trat meist überhaupt keine Flüssigkeit mehr durch. Die für Gradocol-Membranen gültige Regel ${ }^{6}$, mindestens $5 \mathrm{~cm}^{3}$ Lösung pro $\mathrm{cm}^{2}$ Filterfläche passieren zu lassen, um so namentlich anfangs stattfindende Adsorptionen zu eliminieren, ließ sich deshalb nicht einhalten.

Die in Abb. 1 dargestellten Kurven, deren Verlauf bei mehreren, jeweils mit gleichporigen Filtern durchgeführten Einzelversuchen zwar etwas variierte,

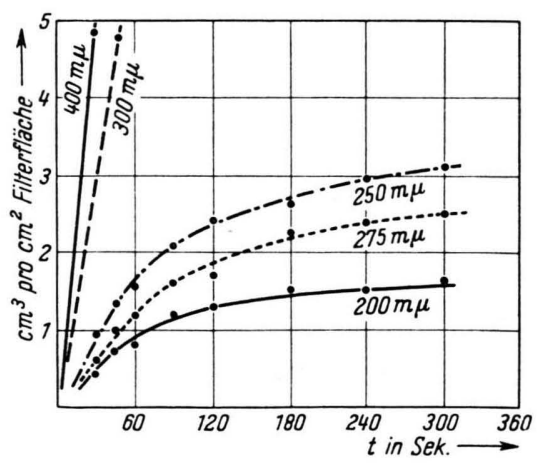

Abb. 1. Filtrations-Geschwindigkeit 1-proz. MäusegehirnHomogenate durch Membranfilter bei 2 Atm. Überdruck.

im Prinzip jedoch stets das gleiche Bild lieferte, veranlaßte uns, außer Adsorptionen vor allem den Einfluß mechanischer Blockierungen zu überprüfen. Bei

10 H. Bechrold u. M. Schlesinger, Biochem. Z. 236, 387 [1931].

11 H. BEchнold, Kolloid-Z. 51, 134 [1930]. diesen Kontrolluntersuchungen, die mit 1-proz. Virus-Suspensionen an Filtern von 600,300 und $275 \mathrm{~m} \mu$ mittlerer Porenweite vorgenommen wurden, bestimmten wir die $L D_{50}$-Werte sukzessiver Filtratproben. Die in Tab. 1 zusammengestellten Ergebnisse zeigen, daß in unserem System keine nennenswerten Adsorptionen vorhanden sind. $\mathrm{Zu}$ gleichen Befunden gelangten Kellenberger et al. ${ }^{12}$ bei der Filtration globulärer Bakterienformen durch Membranfilter. Bei Filtern mit Porenweiten von $300 \mathrm{~m} \mu$ und mehr spielen mechanische Blockierungsvorgänge bis zu Filtratmengen von $5 \mathrm{bzw} .10 \mathrm{~cm}^{3}$ pro $\mathrm{cm}^{2}$ Membranfläche gleichfalls keine Rolle. Ein solches Ergebnis war entsprechend dem geradlinigen Kurvenverlauf der Filtrations-Geschwindigkeiten zu erwarten. Dagegen kommt es bei der Filtration durch Porenweiten unter $300 \mathrm{~m} \mu$ von einem bestimmten Punkt ab zu einer allmählichen Abnahme der Virusaktivität in den Filtraten. Diese Aktivitätsverminderung geht der sich verzögernden Filtrations-Geschwindigkeit etwa parallel und muß auf eine mechanische Blockierung der Membranen zurückgeführt werden.

Den Ergebnissen dieser Vorversuche entsprechend wurden für die Filtrations-Endpunktbestimmung bei Filtern unter $300 \mathrm{~m} \mu$ mittlerer Porenweite jeweils nur $1 \mathrm{~cm}^{3}$ Lösung pro $\mathrm{cm}^{2}$ Filterfläche filtriert.

\section{b) Bestimmung des Filtrations-Endpunktes}

Nach dem Prinzip der „direkten“ Filtration wurden 1-proz. Virus-Suspensionen zunächst bei $600 \mathrm{~m} \mu$ vorfiltriert und anschließend Einzelproben dieses Stammfiltrates getrennt durch die übrigen Membranen geschickt. Von einer fortlaufenden Serienfiltration, bei der das jeweils vorhergehende Filtrat durch das nächstfolgende (engporigere) Filter passiert wird, haben wir abgesehen, da hierdurch das Bild der Filtrationskurve durch die sukzessiven Verluste in jedem Filter verfälscht werden kann.

In Abb. 2 sind die Infektionstiter der Filtrate gegen die mittleren Porenweiten aufgetragen. Die über einen weiten Bereich hin horizontal verlaufende und dann steil abfallende Kurve läßt auf eine relativ homogene Größenverteilung der infektiösen Teilchen schließen. Der Filtrations-Endpunkt liegt bei

12 E. Kellenberger, K. Liebermeister u. V. Bonifas, Z. Naturforschg. 11 b, 206 [1956]. 
etwa $175 \mathrm{~m} \mu$. Unter der Annahme kugelförmiger oder annähernd kugelförmiger Partikel ergibt sich gemäß der Elf or d schen Umrechnungsformel * eine Größenordnung des JHM-Virus von 90 bis $130 \mathrm{~m} \mu$ Durchmesser.

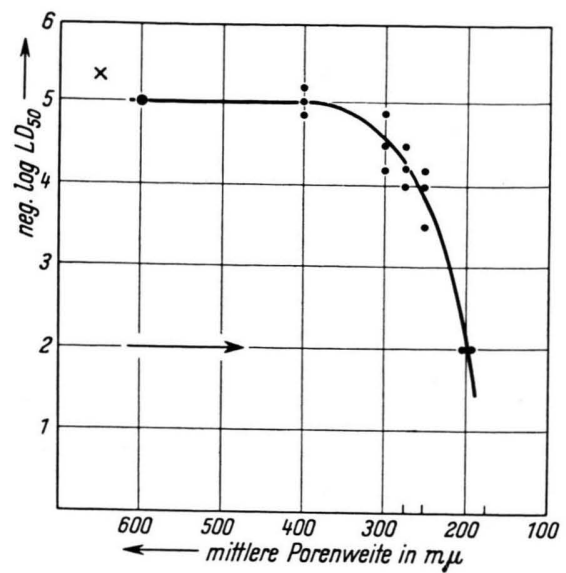

Abb. 2. Filtrationskurve für das JHM-Virus (Einzelheiten s. Text).

Bei gleicher Versuchsanordnung fanden wir ${ }^{13}$ für das A ujeszky-Virus und einen neurotropen Stamm des Herpes simplex-Virus Filtrations-Endpunkte von etwa $275 \mathrm{~m} \mu$, d. h. Größenordnungen zwischen 140 bis $200 \mathrm{~m} \mu$ Durchmesser. Diese Befunde, die als vergleichendes Kriterium unseres Versuchssystems gelten mögen, liegen im Bereich der bislang für beide Virusarten vorliegenden Größenangaben ${ }^{14-17}$.

Ergänzende Versuche mit Viruspräparaten, die durch Kombination von $p_{\mathrm{H}}$-Fällungen und präparativer Ultrazentrifugation partiell gereinigt und angereichert waren (methodische Einzelheiten der Aufarbeitung sind unserer ersten Mitt. zu entnehmen), sollten klären, ob sich die Lage des Filtrations-Endpunktes bei höheren Viruskonzentrationen u. U. in Richtung kleinerer Porenweiten verschiebt. Die Frage konnte jedoch nicht beantwortet werden, da eine schwach geneigte, von 600 bis $200 \mathrm{~m} \mu$ allmählich abfallende Kurve mit einem bei etwa $200 \mathrm{~m} \mu$ gelegenen Filtrations-Endpunkt resultierte. Der Kurvenverlauf weist auf eine heterogene Größenvertei-

* Nach Elford ${ }^{6}$ beträgt der Teilchendurchmesser etwa $1 / 2$ bis $3 / 4$ des Filtrations-Endpunktes, wenn dieser zwischen 100 und $500 \mathrm{~m} \mu$ mittlerer Porenweite gelegen ist.

13 O. Drees u. B. v. KerekJarto, unveröffentlicht.

14 W. J. Elford, J. R. Perdrau u. W. Smith, J. Pathol. Bacteriol. 36, 49 [1933]. lung des infektiösen Prinzips hin und erhärtet die in der ersten Mitt. geäußerte Vermutung, daß während des Reinigens und Anreicherns Aggregationen zwischen Virus untereinander bzw. Virus und Begleitstoffen stattfinden.

\section{Sedimentations-Versuche}

Das Ziel der folgenden Untersuchungen ist eine biologische Bestimmung der Sedimentations-Konstante des JHM-Virus. Zu diesem Zweck wurden 1-proz., in $m / 15$-Phosphatpuffer $\left(p_{\mathrm{H}} 7,4\right)$ hergestellte und niedertourig (15 Min. bei $5000 \mathrm{U} / \mathrm{Min}$.) vorzentrifugierte Virus-Suspensionen in einer „improvisierten Separationszelle“ (vgl. S. 396) bei 8000 und $12000 \mathrm{U} / \mathrm{Min}$. zu jeweils gestaffelten Versuchszeiten ultrazentrifugiert und die dann abpipettierten Überstände auf ihre Infektiosität hin austitriert. Die Titerdifferenzen zwischen dem Ausgangsmaterial und den zentrifugierten Proben liefern einen Maßstab für die während der Zentrifugation erfolgende Konzentrations-Abnahme, aus der die Sedimentations-Konstante nach folgender, von Svedberg und Pedersen ${ }^{18}$ abgeleiteter Formel errechnet werden kann:

$$
\mathrm{s}=-\frac{1}{2 \omega^{2} t} \cdot \ln \left[\frac{x_{0}{ }^{2}}{x_{\mathrm{i}}{ }^{2}}+\frac{C_{\mathrm{t}} \cdot\left(x \mathrm{i}^{2}-x_{0}{ }^{2}\right)}{C_{0} \cdot x_{\mathrm{i}}{ }^{2}}\right],
$$

( $\omega=$ Winkelgeschwindigkeit,,$t=$ Zentrifugierdauer, $x_{0}=$ Abstand zwischen Drehzentrum und Meniskus, $x_{\mathrm{i}}=$ Abstand zwischen Drehzentrum und Zellenboden, $C_{0}=$ Viruskonzentration im Ausgangsmaterial, $C_{t}=$ Viruskonzentration in dem herauspipettierten und durchmischten Zellvolumen nach der Zentrifugation).

Bevor wir uns den Ergebnissen dieser Untersuchungen zuwenden, seien kurz vorangegangene Modellversuche mit EMC- und Poliomyelitis-Viren ${ }^{8}$ gestreift. Hierbei zeigte sich, daß die aus der Abnahme der biologischen Aktivität errechneten Sedimentations-Konstanten befriedigend mit den Werten optischer Messungen anderer Autoren übereinstimmten, solange nur solche Versuche ausgewertet wurden, die hinsichtlich der Zentrifugierdauer jenen Punkt, an dem eine durchschnittliche Aktivitätsabnahme von

15 C. Levaditi, M. Paic u. D. Krassnoff, C. R. Soc. Biol. Filiales Associées 121, 805 [1936].

16 H. Bechrold u. M. Schlesinger, Z. Hyg. Infektionskrankh. 115, 342 [1933].

17 W. J. Elford u. I. A. Galloway, J. of Hyg. 36, 536 [1936].

18 T. Svedberg u. K. O. Pedersen, Die Ultrazentrifuge, Steinkopf, Dresden u. Leipzig 1940. 
etwa $90 \%$ erreicht war, nicht überschritten. Versuche im Bereich jenseits dieser Grenze lieferten infolge gewisser, auch nach langen Zentrifugierzeiten immer noch nachweisbarer „Restaktivitäten“ zunehmend kleiner werdende, von den Ergebnissen optischer Messungen erheblich abweichende SedimentationsKonstanten.

Nach diesen Erfahrungen wollen wir, um mögliche Fehlerquellen bei der anschließenden Auswertung unserer Versuche zu umgehen, zunächst das Verhalten des JHM-Virus während der Ultrazentrifugation näher analysieren. Zu diesem Zweck haben wir aus Titrationsergebnissen die relativen Virus-Konzentrationen der Überstände nach der Zentrifugation errechnet und diese Werte im halblogarithmischen Koordinatensystem gegen die Zentrifugierzeiten aufgetragen (Abb. 3). Die sich daraus ergebenden Kon-
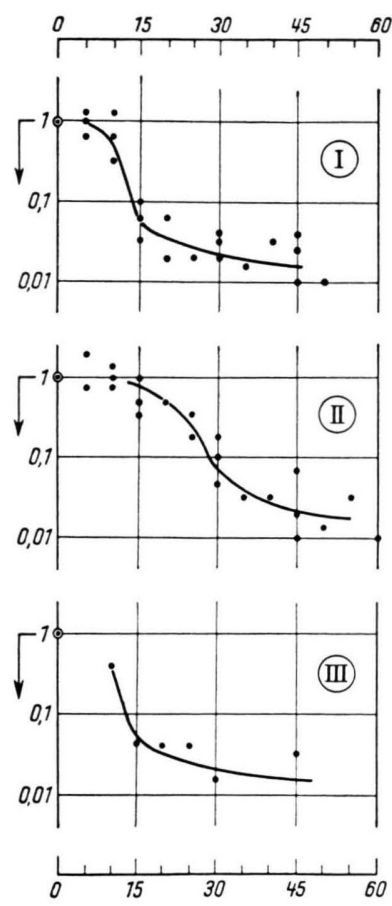

Abb. 3. Aktivitätsabnahme bei der Ultrafiltration des JHMVirus in der „improvisierten Separationszelle“. (Ordinate= relative Viruskonzentration, Abszisse $=$ Zentrifugierdauer in Min.). I: Versuche bei $12000 \mathrm{U} / \mathrm{Min}$. II: Versuche bei 8000 U/Min. III : Versuche bei 12000 U/Min. mit virushaltigen Überständen vorangegangener Ultrazentrifugationen (Einzelheiten hierzu s. Text).

zentrationskurven zeigen einen ähnlichen Verlauf wie diejenigen der oben genannten Modellversuche mit kleinen neurotropen Viren: Sie fallen anfänglich zunehmend steiler $a b$, gehen aber, nachdem eine durchschnittliche Aktivitätsabnahme von etwa $90 \%$ erreicht ist, in einen fast parallel zur Abszisse auslaufenden Abschnitt über. In ergänzenden Versuchen wurden mehrere Proben 20 Min. lang bei $12000 \mathrm{U} /$ Min. zentrifugiert und die etwa 5\% der Ausgangsaktivität enthaltenen Überstände gesammelt. Dieses Material zentrifugierten wir ein zweites Mal zu gestaffelten Zeiten bei 12000 U/Minute. Eine graphische Darstellung der Ergebnisse (Abb. 3, III) liefert das gleiche Bild wie einmalige Zentrifugationen (Abb. 3, I). Daraus geht hervor, daß der horizontal auslaufende Abschnitt der Konzentrationskurven auf „Restaktivitäten“ beruht, die nicht auf etwaige kleinere infektiöse Einheiten, sondern auf methodisch bedingte Faktoren (z. B. geringfügiges Aufwirbeln von bereits sedimentiertem Virus, Anhaften von Virus an den Zellwänden usw.) zurückzuführen sind.

\begin{tabular}{|c|c|c|c|}
\hline & \multicolumn{3}{|c|}{ neg. $\log L D_{50}$} \\
\hline mittl. Porenweite $[\mathrm{m} \mu]$ & 600 & 300 & 275 \\
\hline $\begin{array}{l}\text { Filtratprobe }\left(\mathrm{cm}^{3}\right. \\
\text { pro } \mathrm{cm}^{2} \text { Filterfl.) }\end{array}$ & & & \\
\hline $0-0,5$ & 5,0 & 4,7 & 4,3 \\
\hline $0,5-1,0$ & 5,2 & 4,3 & 4,5 \\
\hline $1,0-1,5$ & 4,8 & 4,7 & 3,5 \\
\hline $1,5-2,0$ & 5,3 & 4,5 & 3,3 \\
\hline $2,0-3,0$ & 5,0 & 4,5 & 2,5 \\
\hline $3,0-5,0$ & 5,2 & 4,3 & - \\
\hline $5,0-10,0$ & 4,8 & - & - \\
\hline
\end{tabular}

Tab. 1. Infektiosität sukzessiver Filtratproben beim Filtrieren 1-proz. Virus-Gehirn-Homogenate durch Membranfilter.

Für das Berechnen der Sedimentations-Konstante kommen somit nur solche Versuche in Betracht, die hinsichtlich der Zentrifugierdauer den Zeitpunkt, an dem eine durchschnittliche Aktivitätsabnahme von etwa $90 \%$ erreicht ist, nicht überschreiten. Da die begrenzte Güte des biologischen Tests* die Zuverlässigkeit der errechneten Sedimentations-Konstanten naturgemäß um so mehr beeinträchtigt, je kleiner das Verhältnis $C_{\mathrm{t}} / C_{0}$ ist, haben wir bei der Auswertung ferner auf jene Versuche verzichtet, die im Bereich einer durchschnittlichen Aktivitätsabnahme von weniger als $70 \%$ liegen. In Abb. 2 sind alle verwertbaren Experimente und die daraus errechneten Sedimentations-Konstanten zusammengestellt. Die Einzelwerte schwanken bei einer Drehzahl von $12000 \mathrm{U} /$ Min. zwischen 1060 und $1340 \mathrm{~S}$, bei 8000

* Bei unseren Versuchen streuten die Infektionstiter im Bereich des Wertes log 0,5 . 


\begin{tabular}{|c|c|c|c|c|c|c|c|c|}
\hline \multirow{2}{*}{ C/Min. } & \multirow{2}{*}{$\begin{array}{c}\text { Zentrifugierdauer } \\
\text { [Sek.] }\end{array}$} & \multicolumn{2}{|c|}{$-\log L D_{50}{ }^{*}$} & \multirow{2}{*}{$\underset{\text { Titerdifferenz }}{\log }$} & \multirow{2}{*}{$C_{t} / C_{0}$} & \multirow{2}{*}{$s_{20}$} & \multicolumn{2}{|c|}{$\begin{array}{c}\text { Teilchengröße } \\
(\mathrm{m} \mu \varnothing)\end{array}$} \\
\hline & & $v$ & $n$ & & & & $d=1,1^{* *}$ & $d=1,3 * *$ \\
\hline 12.000 & $\begin{array}{r}900 \\
900 \\
900 \\
1200 \\
1200\end{array}$ & $\begin{array}{l}5,0 \\
4,8 \\
5,0 \\
5,0 \\
4,7\end{array}$ & $\begin{array}{l}4,0 \\
3,5 \\
3,5 \\
3,7 \\
3,0\end{array}$ & $\begin{array}{l}1,0 \\
1,3 \\
1,5 \\
1,3 \\
1,7\end{array}$ & $\begin{array}{l}0,1 \\
0,05 \\
0,03 \\
0,05 \\
0,02\end{array}$ & $\begin{array}{l}1230 \\
1290 \\
1340 \\
1060 \\
1150\end{array}$ & & \\
\hline \multicolumn{6}{|c|}{ Mittelwert } & 1214 & 152 & 86 \\
\hline 8.000 & $\begin{array}{l}1500 \\
1500 \\
1800 \\
1800 \\
1800 \\
2100\end{array}$ & $\begin{array}{l}5,0 \\
4,7 \\
4,7 \\
5,0 \\
5,0 \\
5,3\end{array}$ & $\begin{array}{l}4,0 \\
4,0 \\
4,0 \\
4,0 \\
3,7 \\
1,8\end{array}$ & $\begin{array}{l}0,5 \\
0,7 \\
0,7 \\
1,0 \\
1,3 \\
1,5\end{array}$ & $\begin{array}{l}0,32 \\
0,20 \\
0,20 \\
0,10 \\
0,05 \\
0,03\end{array}$ & $\begin{array}{r}735 \\
1120 \\
880 \\
1020 \\
1070 \\
990\end{array}$ & & \\
\hline \multicolumn{6}{|c|}{ Mittelwert } & 969 & 135 & 77 \\
\hline
\end{tabular}

Tab. 2. Biologisch bestimmte Sedimentations-Konstanten und daraus errechnete Teilchengröße des JHM-Virus.

U/Min. zwischen 735 und 1120 S. Die entsprechenden Mittelwerte betragen 1214 und 969 S.

Um einen Vergleich zwischen den Ergebnissen der Ultrazentrifugation und denjenigen der Ultrafiltration anstellen zu können, haben wir aus den Mittelwerten der Sedimentations-Konstante die Teilchengröße des JHM-Virus nach der modifizierten S tokes-schen Gleichung

$$
r=\sqrt{\frac{9}{2} \frac{\mathrm{s}_{20} \cdot \eta}{\varrho \mathrm{V}-\varrho \mathrm{MI}}}
$$

errechnet $\quad(r=$ Teilchenradius, $\eta=$ Viskosität von Wasser bei $20^{\circ} \mathrm{C}, \varrho_{\mathrm{M}}=$ Dichte des Mediums (Phosphatpuffer), $\varrho_{\mathrm{V}}=$ Dichte des Virus). Da die Dichte des Virus nicht bestimmt werden konnte, wurden den Berechnungen die beiden Grenzwerte $\varrho=1,1$ und $\varrho=1,3$ zugrundegelegt. Für $\mathrm{s}_{20}=1214$ ergaben sich Teilchendurchmesser von 152 und $86 \mathrm{~m} \mu$, für $\mathrm{s}_{20}=969$ solche von 135 und $77 \mathrm{~m} \mu$ (s. Tab. 2, vorletzte und letzte Spalte).

\section{Besprechung der Ergebnisse}

Das JHM-Virus ist nach den Ergebnissen unserer Untersuchungen in die Gruppe der mittelgroßen tierpathogenen Virusarten einzuordnen. Der steile Abfall der Filtrationskurve läßt auf eine relativ homogene Größenverteilung und eine kugelförmige oder zumindest annähernd kugelförmige Gestalt schließen. Für die Annahme, daß das Achsenverhältnis der Viruspartikel nicht wesentlich von dem einer Kugel abweicht, spricht ferner die befriedigende Úbereinstimmung der aus den Filtrations- und Sedimentations-Versuchen ermittelten Teilchendurchmesser. Im übrigen lehren die Ergebnisse der Ultrazentrifugation, daß mindestens 99,9\% des infektiösen Prinzips einer Fraktion zuzuordnen sind, deren Sedimentations-Konstante zu etwa $1000 \mathrm{~S}$ bestimmt werden konnte. $\mathrm{Ob}$ innerhalb dieser Fraktion eine strenge Einheitlichkeit hinsichtlich der Teilchengröße und der Sedimentations-Konstante besteht, läßt sich infolge der begrenzten Güte biologischer Untersuchungsmethoden nicht sagen.

Abschließend sei betont, daß die aus den vorliegenden Untersuchungen errechneten Teilchendurchmesser und Sedimentations-Konstanten den Wert einer ersten Annäherung nicht übertreffen. Die exaktere Analyse physikalischer Eigenschaften setzt eine uns bislang nicht gelungene Adaptation des Virus an Wirtssysteme voraus, die höhere Virusausbeuten als Mäusegehirne ermöglichen. Genauere Angaben über die morphologischen Strukturen des Virus müssen elektronenoptischen Untersuchungen, die von uns inzwischen begonnen wurden, vorbehalten bleiben.

Dem Verein zur Förderung der Erforschung und Bekämpfung der spinalen Kinderlähmung (Bielefeld) möchten wir auch an dieser Stelle für die Bereitstellung der Phywe-Ultrazentrifuge danken. 